\title{
PRICING OF THE AMERICAN PUT UNDER LÉVY PROCESSES
}

\author{
SERGEI LEVENDORSKII
}

\author{
Rostov State University of Economics, \\ B. Sadovaya 69, Rostov-on-Don, 344007, Russia \\ e-mail: leven@rnd.runnet.ru
}

\begin{abstract}
We consider the American put with the finite time horizon, $T$, assuming that under a chosen equivalent martingale measure stock returns follow a regular Lévy process of exponential type. We formulate the free boundary value problem for the price of the American put, and develop the non-Gaussian analog of the method of lines and Carr's randomization method used in the Gaussian option pricing theory. The result is the (discretized) early exercise boundary and prices of the American put for all strikes and maturities from 0 to $T$. In the case of exponential jump-diffusion processes, a simple efficient pricing scheme is constructed.
\end{abstract}

Key words: American put, finite time horizon, Lévy processes

\section{INTRODUCTION}

Consider a market of a riskless bond and a stock whose returns follow a non-Gaussian process; then the market is typically incomplete. According to the modern martingale approach to option pricing (see [18] and the bibliography therein), arbitrage-free prices can be obtained as expectations under any equivalent martingale measure (EMM), which is absolutely continuous w.r.t. the historic measure. Let the riskless rate $r>0$ be fixed, let $S=\left\{S_{t}\right\}_{t \geq 0}, S_{t}=\exp X_{t}$, be the price process of the stock, which pays no dividend, and let $\mathbf{Q}$ be an EMM chosen by the market. We assume that under $\mathbf{Q}, X$ is a regular Lévy process of exponential type (RLPE); this general class contains essentially all classes of Lévy processes used in empirical studies of financial markets, jump-diffusions with exponentially distributed jump sizes in particular. The class of RLPE can be loosely characterized as a class of Lévy processes with the Lévy measures exponentially decaying at infinity and having polynomial singularity at the origin. It was introduced in [8][9], under a misleading name Generalized Truncated Lévy Processes. 
The name RLPE was suggested in [5], and in [10]-[12] and the present paper, we use the new name.

Consider the American option, with the terminal payoff $g$ and the expiry date $T$. In $[9,10]$, the pricing problem for perpetual American options is solved by using the Wiener-Hopf factorization method, and the same method is used here (notice that we use the WienerHopf factorization method in the form different from [22]). In the case of the American put with a finite time horizon, explicit formulas are not available even in the Brownian motion (BM) case, and so approximate methods have to be used. We start with the reformulation of the pricing problem as a free boundary value problem, then discretize the time derivative, as in [14] and [13] in the Gaussian case (the analytical method of lines and Carr's randomization method; both methods lead to the same computational procedure). The derivation of pricing procedure for the time-discretized free boundary problem is different from the one in the Gaussian case, and its implementation relies on the formulas for the factors in the Wiener-Hopf factorization formula. Here approximate formulas for the factors obtained in [10] and [12] can be useful; in some cases, for jump-diffusions in particular, the explicit formulas for the factors can be derived. A general form of the pricing procedure for American put was developed in Chapter 6 of [12]. In this paper, we make the procedure more detailed, and in the case of jump-diffusions, derive an explicit simple efficient algorithm. Numerical examples are provided to demonstrate the dependence of the shape of the early exercise boundary and American put price on positive and negative jumps.

For other methods of pricing of American options in the Gaussian case, see e.g. [36], [32], [25], [23], [27], [16] and the bibliography therein.

The rest of the paper is organized as follows. In Section 2, the model classes of RLPE are described, and the general definition of RLPE is introduced. In Section 3, explicit formulas for the factors in the WienerHopf factorization formula, with examples, are provided. In Section 4, we formulate the free boundary problem for the American put, under a general RLPE, consider the time discretization of the problem, recall a general pricing scheme from Section 6.2 of [12], and analyze it further. In Section 5, we consider the case of jump-diffusions, and construct an explicit simple pricing procedure. In Section 6 , we provide a detailed algorithm for a general RLPE. Section 7 concludes. 


\section{Regular LÉvy processes of exponential type}

2.1. General definitions. Recall that a Lévy process is a process with stationary independent increments (for general definitions, see e.g. [34]). It may have a Gaussian component and/or pure jump component. The latter is characterized by the density of jumps, which is called the Lévy density. We denote it by $F(d x)$. A Lévy process can be completely specified by its characteristic exponent, $\psi$, definable from the equality $E\left[e^{i \xi X(t)}\right]=e^{-t \psi(\xi)}$ (we confine ourselves to the one-dimensional case). If the jump component is a compound Poisson process then the characteristic exponent is given by

$$
\psi(\xi)=-i b \xi+\frac{\sigma^{2}}{2} \xi^{2}+\int_{-\infty}^{+\infty}\left(1-e^{i \xi y}\right) F(d y)
$$

where $\sigma^{2}$ and $b$ are the variance and drift coefficient of the Gaussian component, and $F(d y)$ satisfies

$$
\int_{\mathbf{R} \backslash\{0\}} \min \{1,|y|\} F(d y)<+\infty .
$$

Equation (2.1) is a special case of the Lévy-Khintchine formula; for the general case, see e.g. [34].

Let $\lambda_{-}<0<\lambda_{+}$and $\nu \in(0,2]$. A Lévy process is called a Regular Lévy Process of exponential type $\left[\lambda_{-}, \lambda_{+}\right]$and order $\nu>0$ if the following two conditions are satisfied:

a) the characteristic exponent admits a representation

$$
\psi(\xi)=-i \mu \xi+\phi(\xi)
$$

where $\phi$ is holomorphic ${ }^{1}$ in the strip $\Im \xi \in\left(\lambda_{-}, \lambda_{+}\right)$, continuous up to the boundary of the strip, and admits a representation

$$
\phi(\xi)=c|\xi|^{\nu}+O\left(|\xi|^{\nu_{1}}\right)
$$

as $\xi \rightarrow \infty$ in the strip $\Im \xi \in\left[\lambda_{-}, \lambda_{+}\right]$, where $\nu_{1}<\nu$;

b) there exist $\nu_{2}<\nu$ and $C$ such that the derivative of $\phi$ in (2.2) admits a bound

$$
\left|\phi^{\prime}(\xi)\right| \leq C(1+|\xi|)^{\nu_{2}}, \quad \Im \xi \in\left[\lambda_{-}, \lambda_{+}\right] .
$$

One can easily generalize this definition by using $c_{ \pm} \geq 0$ in (2.3), as $\Re \xi \rightarrow \pm \infty$.

\footnotetext{
${ }^{1}$ A function $f$ is said to be holomorphic, or analytic, on an open subset $U$ of the complex plane $\mathbf{C}$, iff $f^{\prime}(\xi)$ exists for all $\xi \in U$.
} 
2.2. Examples. Wide families of jump-diffusion processes used in the theoretical and empirical studies of financial markets are RLPE of order 2 .

Example 2.1. Let $X$ be a Lévy process with the Lévy density

$$
F(d x)=c_{+} \lambda_{+} e^{\lambda_{+} x} \mathbf{1}_{(-\infty, 0)}(x) d x+c_{-}\left(-\lambda_{-}\right) e^{\lambda_{-} x} \mathbf{1}_{(0,+\infty)}(x) d x,
$$

where $\lambda_{+}>0, \lambda_{-}<-1$ and $c_{ \pm}>0$. Then

$$
\psi(\xi)=\frac{\sigma^{2}}{2} \xi^{2}-i b \xi+\frac{i c_{+} \xi}{\lambda_{+}+i \xi}+\frac{i c_{-} \xi}{\lambda_{-}+i \xi},
$$

where $\sigma^{2}>0$ and $b \in \mathbf{R}$ are the variance and drift of the Gaussian component. The $\psi(\xi)$ is holomorphic in the strip $\Im \xi \in\left(\lambda_{-}, \lambda_{+}\right)$.

The following classes of Lévy processes without the gaussian component and with exponentially decaying tails of probability densities have been widely used to describe the behavior of stock prices in real financial markets, and almost all of them are RLPE's:

Variance Gamma Processes have been used by Madan and co-authors in a series of papers during 90th (see [28] and the bibliography there);

Hyperbolic Processes were constructed and used by Eberlein and co-authors [19], [20], [21]; hyperbolic distributions were constructed in [2];

Normal Inverse Gaussian processes (NIG) were constructed in [3] and used to model German stocks in [4]; generalization of the class NIG, namely, the class of Normal Tempered Stable Lévy Processes, was constructed in [5] and [6].

Example 2.2. The characteristic exponent of a NIG is of the form

$$
\psi(\xi)=-i \mu \xi+\delta\left[\left(\alpha^{2}-(\beta+i \xi)^{2}\right)^{1 / 2}-\left(\alpha^{2}-\beta^{2}\right)^{1 / 2}\right],
$$

where $\nu \in(0,2), \delta>0$, and $\alpha>|\beta|$; it is holomorphic in the strip $\Im \xi \in(-\alpha-\beta, \alpha-\beta)$, and (2.2)-(2.4) are satisfied in this strip, with $\nu=1$.

Truncated Lévy Processes (TLP) constructed by Koponen[24] were used for modeling in real financial markets in [7], [17] and [31]; a generalization of this family was constructed in [8], [9]. Later, this generalization was used in [15] under the name CGMY-model. As A.N. Shiryaev remarked, the name TLP was misleading, and so starting with [10]-[11] we call this family of processes KoBoL family.

Example 2.3. The characteristic exponent of a process of KoBoL family is of the form

$$
\psi(\xi)=-i \mu \xi+c \Gamma(-\nu)\left[\lambda_{+}^{\nu}-\left(\lambda_{+}+i \xi\right)^{\nu}+\left(-\lambda_{-}\right)^{\nu}-\left(-\lambda_{-}-i \xi\right)^{\nu}\right],
$$

where $\nu \in(0,2), \nu \neq 1, c>0, \lambda_{-}<0<\lambda_{+}$, and $\mu \in \mathbf{R}$; it is holomorphic in a strip $\Im \xi \in\left(\lambda_{-}, \lambda_{+}\right)$, and (2.2)-(2.3) are satisfied in this strip. 
For $\nu \in(0,1)$, the equation (2.8) is obtained from (2.1) with $F(d x)=c \lambda_{+} e^{\lambda_{+} x} \mathbf{1}_{(-\infty, 0)}(x)|x|^{-\nu-1} d x+c\left(-\lambda_{-}\right) e^{\lambda_{-} x} \mathbf{1}_{(0,+\infty)}(x) x^{-\nu-1} d x$, and $\sigma=0$ (that is, there is no Gaussian component). In the case $\nu \in(1,2)$, instead of $(2.1)$, the general form of the Lévy-Khintchine formula is needed (see [12]).

Earlier, non-infinitely divisible truncations of stable Lévy distributions had been constructed and used to model the behavior of the Standard \& Poor 500 Index by Mantegna and Stanley [29], [30].

Notice that BM, HP, NIG, NTS Lévy processes are RLPE, and any finite mixture of independent BM, NIG, HP and TLP are RLPE. Variance Gamma Processes (VGP) are excluded, since they need special treatment at many places; in particular, the explicit formulas for the factors in the Wiener-Hopf factorization formula, which is used here, need regularization in the case of VGP.

2.3. The infinitesimal generator of a Lévy process. If $X$ is a Lévy process on $\mathbf{R}$, with the characteristic exponent given by (2.1), then its infinitesimal generator, denote it $L$, is an integro-differential operator which acts as follows:

$$
L u(x)=\frac{\sigma^{2}}{2} u^{\prime \prime}(x)+b u^{\prime}(x)+\int_{-\infty}^{+\infty}(u(x+y)-u(x)) F(d y)
$$

for the formula in the general case, see e.g. [34, 12]. The generator of a Lévy process in $\mathbf{R}^{n}$ with the characteristic exponent $\psi$ can be represented in the form $L=-\psi(D)$, that is, in the form of a pseudodifferential operator (PDO) with the symbol $-\psi$ (See e.g. [10]-[12]). Recall that a pdo $A=a(D)$ acts as follows:

$$
A u(x)=(2 \pi)^{-n} \int_{\mathbf{R}^{n}} e^{i\langle x, \xi\rangle} a(\xi) \hat{u}(\xi) d \xi,
$$

where $\hat{u}$ is the Fourier transform of a function $u$ :

$$
\hat{u}(\xi)=\int_{\mathbf{R}^{n}} e^{-i\langle x, \xi\rangle} u(x) d x
$$

\section{WIENER-HOPF FACTORIZATION}

3.1. General Lévy processes. Let $(\Omega, \mathcal{F}, \mathbf{P})$ be a probability space, on which a one-dimensional Lévy process $X$ is defined, and let $\Omega_{0}$ be a subset of $\Omega$ such that for each $\omega \in \Omega_{0}$, the trajectory $X .(\omega)$ is right-continuous with left limits. On $\Omega_{0}$, define $M_{t}=\sup _{0 \leq s \leq t} X_{s}$ and $N_{t}=\inf _{0 \leq s \leq t} X_{s}$. On $\Omega \backslash \Omega_{0}$, both $M_{t}$ and $N_{t}$ are set to be 0 . $M=\left\{M_{t}\right\}$ and $N=\left\{N_{t}\right\}$ are called the supremum process and the 
infimum process, respectively. The Laplace transform (in $t$ ) of the distribution of $X_{t}$, or more precisely,

$$
q E^{x}\left[\int_{0}^{\infty} e^{-q t} e^{i \xi X_{t}} d t\right]=q(q+\psi(\xi))^{-1},
$$

can be factorized by using the Laplace transforms (in $t$ ) of the distributions of the supremum and infimum processes. Namely, let $T$ be the exponentially distributed random variable on $[0,+\infty)$ with the mean $q^{-1}$. Introduce functions

$$
\phi_{q}^{+}(\xi)=E\left[e^{i \xi M_{T}}\right], \quad \phi_{q}^{-}(\xi)=E\left[e^{i \xi N_{T}}\right] .
$$

Among many factorization identities, we will use only the simplest one (see e.g. [33], p.89, or [34], Theorems 45.2 and 45.5; for more detailed exposition, see [34], Section 45).

Theorem 3.1. (i) Let $q>0$. Then $\phi_{q}^{ \pm}(\xi)$ are the Fourier transforms of infinitely divisible distributions $P_{q}^{+}$and $P_{q}^{-}$supported on $(-\infty, 0]$ and $[0,+\infty)$, respectively:

$$
\begin{aligned}
& \phi_{q}^{+}(\xi)=\int_{-\infty}^{+\infty} e^{-i x \xi} P_{q}^{+}(d x)=\int_{-\infty}^{0} e^{-i x \xi} P_{q}^{+}(d x), \\
& \phi_{q}^{-}(\xi)=\int_{-\infty}^{+\infty} e^{-i x \xi} P_{q}^{-}(d x)=\int_{0}^{+\infty} e^{-i x \xi} P_{q}^{-}(d x)
\end{aligned}
$$

(ii) the following factorization identity holds:

$$
q(q+\psi(\xi))^{-1}=\phi_{q}^{+}(\xi) \phi_{q}^{-}(\xi), \quad \xi \in \mathbf{R} .
$$

Notice that $\phi_{q}^{+}(\xi)$ (resp., $\left.\phi_{q}^{-}(\xi)\right)$ admits the analytic continuation into the upper half-plane $\Im \xi>0$ (resp., lower half-plane $\Im \xi<0$ ) and does not vanish there. Thus, (3.4) is a special case of the Wiener-Hopf factorization introduced in solving integral equations by Wiener and Hopf in 1931 [35], and widely used in Queuing theory and Insurance. In [22] and Chapter 11 in [12], it is applied to problems of endogenous default.

Formulas (3.1) are by no means explicit though very convenient for theoretical considerations. There are also general formulas, which are rather involved (see e.g. [34], Theorem 45.2). Simple explicit analytical formulas can be obtained for special cases only.

3.2. Lévy processes of exponential type. We fix a branch of ln by the requirement $\ln a \in \mathbf{R}$ for $a>0$. The following theorem is a special case of Theorem 3.2 in [12]. 
Theorem 3.2. Let $X$ be an RLPE, and let there exist $c_{1}>0$ and $\omega_{-}<0<\omega_{+}$such that

$$
\Re \psi(\xi) \geq c_{1}, \quad \Im \xi \in\left[\omega_{-}, \omega_{+}\right] .
$$

Then

a) $\phi_{q}^{+}(\xi)$ admits the analytic continuation into a half-plane $\Im \xi>\omega_{-}$ and can be calculated as follows:

$$
\begin{aligned}
\phi_{q}^{+}(\xi) & =\exp \left[(2 \pi i)^{-1} \int_{-\infty+i \omega_{-}}^{+\infty+i \omega_{-}} \frac{\psi^{\prime}(\eta)}{q+\psi(\eta)} \ln \frac{\eta-\xi}{\eta} d \eta\right] \\
& =\exp \left[(2 \pi i)^{-1} \int_{-\infty+i \omega_{-}}^{+\infty+i \omega_{-}} \frac{\xi \ln (q+\psi(\eta))}{\eta(\xi-\eta)} d \eta\right]
\end{aligned}
$$

b) $\phi_{q}^{-}(\xi)$ admits the analytic continuation into a half-plane $\Im \xi<\omega_{+}$ and can be calculated as follows:

$$
\begin{aligned}
\phi_{q}^{-}(\xi) & =\exp \left[-(2 \pi i)^{-1} \int_{-\infty+i \omega_{+}}^{+\infty+i \omega_{+}} \frac{\psi^{\prime}(\eta)}{q+\psi(\eta)} \ln \frac{\eta-\xi}{\eta} d \eta\right] \\
& =\exp \left[-(2 \pi i)^{-1} \int_{-\infty+i \omega_{+}}^{+\infty+i \omega_{+}} \frac{\xi \ln (q+\psi(\eta))}{\eta(\xi-\eta)} d \eta\right]
\end{aligned}
$$

By using formulas (3.6)-(3.9), it is possible to calculate the factors in the Wiener-Hopf factorization numerically. In some cases, for KoBoL and NIG in particular, the numerical procedure can be made fairly efficient by reducing the integration to an appropriate cut in the complex plane (this reduction was used in [10] and Chapters 5 and 12 of [12]).

3.3. Jump-diffusion case. The most simple formulas can be obtained in the case of a rational $\psi$, an example being the jump-diffusion process given by (2.6). By using general formulas and the residue theorem, it is straightforward to conclude that the factors $\phi_{q}^{ \pm}(\xi)$ are rational functions of $\xi$ (for details, see e.g. Chapter 13 in [12]). Hence, the factorization of $a(\xi)=q+\psi(\xi)$ can be obtained as follows:

(i) represent $a$ in the form $a=P / Q$, where $P$ and $Q$ are polynomials;

(ii) notice that both $P$ and $Q$ have no roots on the real line, and find the sets of roots of $P,\left\{-i \beta_{j}^{+}(q)\right\}$ and $\left\{-i \beta_{j}^{-}(q)\right\}$, in the lower and upper complex plane, respectively, and the sets of roots of $Q$, $\left\{-i \lambda_{l}^{+}\right\}$and $\left\{-i \lambda_{l}^{-}\right\}$, in the same half-planes; 
(iii) set

$$
\begin{aligned}
\phi_{q}^{+}(\xi) & =\prod_{j} \frac{\beta_{j}^{+}(q)}{\beta_{j}^{+}(q)-i \xi} \prod_{l} \frac{\lambda_{l}^{+}-i \xi}{\lambda_{l}^{+}} \\
\phi_{q}^{-}(\xi) & =\prod_{j} \frac{\beta_{j}^{-}(q)}{\beta_{j}^{-}(q)-i \xi} \prod_{l} \frac{\lambda_{l}^{-}-i \xi}{\lambda_{l}^{-}} .
\end{aligned}
$$

Example 3.1. Consider the jump-diffusion process in Example 2.1. Set $\xi=-i \beta$ and

$$
M(\beta):=\psi(-i \beta)=-b \beta-\frac{\sigma^{2}}{2} \beta^{2}+\frac{c_{+} \beta}{\lambda_{+}+\beta}+\frac{c_{-} \beta}{\lambda_{-}+\beta} .
$$

From (2.6), the equation

$$
q+\psi(\xi)=0
$$

assumes the form

$$
q+M(\beta)=0
$$

Since a polynomial of degree 4 has 4 roots in the complex plane, equation (3.13) has 4 real roots at most. The real-valued function $q+M(\cdot): \mathbf{R} \backslash\left\{-\lambda_{+},-\lambda_{-}\right\} \rightarrow \mathbf{R}$ is continuous on intervals $\left(-\infty,-\lambda_{+}\right)$, $\left(-\lambda_{+}, 0\right),\left(0,-\lambda_{-}\right)$and $\left(-\lambda_{-},+\infty\right)$, and it changes sign on each of these intervals, since $q>0, M(0)=0, M\left(-\lambda_{-}-0\right)=M\left(-\lambda_{+}+0\right)=-\infty$, $M( \pm \infty)=-\infty$, and $M\left(-\lambda_{-}+0\right)=M\left(-\lambda_{+}-0\right)=+\infty$. Hence equation (3.13) has at least one root on each of these intervals. The number of the intervals being 4 , there exists exactly one root on each interval; we call these roots $\beta_{1}^{-}=\beta_{1}^{-}(q), \beta_{0}^{-}=\beta_{0}^{-}(q), \beta_{0}^{+}=\beta_{0}^{+}(q)$, and $\beta_{1}^{+}=\beta_{1}^{+}(q)$, respectively. Thus, (3.10) and (3.11) become

$$
\begin{aligned}
\phi_{q}^{+}(\xi) & =\frac{-\lambda_{-}-i \xi}{-\lambda_{-}} \prod_{j=0,1} \frac{\beta_{j}^{+}}{\beta_{j}^{+}-i \xi} \\
\phi_{q}^{-}(\xi) & =\frac{\lambda_{+}+i \xi}{\lambda_{+}} \prod_{j=0,1} \frac{-\beta_{j}^{-}}{-\beta_{j}^{-}+i \xi} .
\end{aligned}
$$

For a generalization to the multi-jump case, see Section 5.

3.4. Factors $\phi_{q}^{ \pm}(\xi)$ and related operators. Notice that in the case of an RLPE, the distributions $P_{q}^{ \pm}(d x)$ have densities:

$$
P_{q}^{ \pm}(d x)=k_{q}^{ \pm}(x) d x
$$


(see $[10,12])$, and introduce operators $\phi_{q}^{ \pm}(D)$ by

$$
\begin{aligned}
\phi_{q}^{+}(D) u(x) & =\int_{-\infty}^{0} u(x-y) P_{q}^{+}(d y) \\
& =\int_{x}^{+\infty} u(y) k_{q}^{+}(x-y) d y \\
\phi_{q}^{-}(D) u(x) & =\int_{0}^{+\infty} u(x-y) P_{q}^{-}(d y) \\
& =\int_{-\infty}^{x} u(y) k_{q}^{-}(x-y) d y .
\end{aligned}
$$

In the jump-diffusion case, the $k_{q}^{ \pm}$are exponential polynomials, and so the formulas (3.16)-(3.17) are quite easy to apply (see Section 5); in the case of more general RLPE, the densities $k_{q}^{ \pm}$can be found by using the formulas for the factors $\phi_{q}^{ \pm}$and the Fourier inversion:

$$
k_{q}^{ \pm}(x)=(2 \pi)^{-1} \int_{-\infty+i \omega_{\mp}}^{+\infty+i \omega_{\mp}} e^{i x \xi} \phi_{q}^{ \pm}(\xi) d \xi, \quad \pm x<0 .
$$

(Here $\omega_{-}<0<\omega_{+}$are from Theorem 3.2). Notice that the action on exponents is calculated quite easily for any RLPE: if $-i \beta$ belongs to the half-plane, where $\phi_{q}^{ \pm}$is defined, then

$$
\phi_{q}^{ \pm}(D) e^{\beta x}=\phi_{q}^{ \pm}(-i \beta) e^{\beta x} .
$$

In particular, $\phi_{q}^{ \pm}(D) 1=1$.

\section{Approximate pricing of the American put}

4.1. The free boundary value problem. We consider the American put on a stock which pays no dividends; the generalization to the case of a dividend-paying stock and the American call is straightforward. Let $Q$ be an EMM chosen by the market, and let $L=L^{\mathbf{Q}}$ be the infinitesimal generator of $X$ under $\mathbf{Q}$. Assume that the optimal stopping time is of the form $\tau_{B}^{\prime} \wedge T$, where $\tau_{B}^{\prime}$ is the hitting time of a closed set $B \subset \mathbf{R} \times(-\infty, T]$ by the two-dimensional process $\hat{X}_{t}=\left(X_{t}, t\right)$. Set $\mathcal{C}=\mathbf{R} \times[0, T) \backslash B$ (this is the region, where the option remains alive), and consider the following boundary value problem

$$
\begin{aligned}
\left(\partial_{t}+L-r\right) V(x, t) & =0, \quad(x, t) \in \mathcal{C} \\
V(x, t) & =K-e^{x}, \quad(x, t) \in B \text { or } t=T ; \\
V(x, t) & \geq\left(K-e^{x}\right)_{+}, \quad t \leq T, x \in \mathbf{R} \\
\left(\partial_{t}+L-r\right) V(x, t) & \leq 0, \quad t<T, \quad(x, t) \notin \overline{\mathcal{C}} .
\end{aligned}
$$


Under certain regularity conditions (see Theorem 6.1 in [12]), the continuous bounded solution to the free boundary problem (4.1)-(4.4) gives the optimal early exercise region, $B$, and the rational option price, $V_{*}$.

4.2. Analytical method of lines. Carr and Faguet [14] suggested to use the time discretization in the Black-Scholes equation, and obtained semi-explicit analytical formulas for the early exercise boundary and put price. The same formulas can be derived by Carr's randomization procedure [13]. In [14] and [13], the Brownian motion case was studied and the time derivative in the Black-Scholes equation was discretized; in the non-Gaussian situation, we discretize the derivative $\partial_{t}$ in the generalized Black-Scholes equation (4.1). In the Gaussian case, it is possible to use the smooth pasting principle to replace the optimality conditions (4.3)-(4.4). In [10] (see also Chapter 5 in [12]), it is shown in the case of perpetual American put that the smooth pasting principle may fail in the non-Gaussian case, therefore for the American put with the finite time horizon, the optimal discretized boundary should be found by the other means similar to the ones used in [10], in the perpetual American option case.

Below, we recall the pricing procedure developed in Section 6.2.2 of [12]. Divide [0,T] into $n$ subperiods by points $t_{j}=j \Delta, j=0,1, \ldots, n$, where $\Delta=T / n$, and denote by $v_{j}(x)$ the approximation to $V\left(x, t_{j}\right) ; h_{j}$ denotes the approximation to the early exercise boundary at time $t_{j}$. Then $v_{n}(x)=\max \left\{K-e^{x}, 0\right\}$, and by discretizing the derivative $\partial_{t}$ in (4.1), we obtain, for $j=n-1, n-2, \ldots, 0$,

$$
\Delta^{-1}\left(v_{j+1}(x)-v_{j}(x)\right)-(r-L) v_{j}(x)=0, \quad x>h_{j},
$$

or

$$
(1+\Delta(r-L)) v_{j}(x)=v_{j+1}(x), \quad x>h_{j}
$$

Equation (4.2) assumes the form

$$
v_{j}(x)=K-e^{x}, \quad x \leq h_{j} .
$$

Set $q=\Delta^{-1}+r$, and factorize $q+\psi(\xi)$ (see (3.4)). By using the WienerHopf factorization method, it is possible to find the unique bounded continuous solution to the problem (4.5)-(4.6):

$$
v_{j}(x)=K-e^{x}+(1+r \Delta)^{-1} \phi_{q}^{-}(D) \mathbf{1}_{\left[h_{j},+\infty\right)} w_{j}(x),
$$

where

$$
w_{j}=\phi_{q}^{+}(D)\left[v_{j+1}+e^{x}-(1+r \Delta) K\right] .
$$

Lemma 4.1. ([12], Lemma 6.1.) For $j=n-1, \ldots, 0$, the function $w_{j}$ is increasing, and it changes the sign.

From Lemma 4.1, $w_{j}$ has the only zero, call it $h_{j}$. 
Theorem 4.2. ([12], Theorem 6.2). The optimal exercise boundary at time $t_{j}$ is $h_{j}, j=n-1, n-2, \ldots, 0$.

To sum up: the algorithm for the discrete time approximation to the optimal exercise boundary and rational put price is as follows:

1. Set $h_{n}=\ln K, \quad v_{n}(x)=\max \left\{K-e^{x}, 0\right\}$;

2. for $j=n-1, n-2, \ldots$, define $w_{j}$ by $(4.8)$;

3. find $h_{j}$ as the unique solution to the equation

$$
w_{j}(x)=0
$$

4. for $x>h_{j}$, define $v_{j}(x)$ by (4.7).

This general scheme was derived in [12], p.158.

4.3. Further analysis of the scheme. For any $\Delta>0, q+\psi(0)=$ $q>0$, and $q+\psi(-i)=\Delta^{-1}+r+\psi(-i)=\Delta^{-1}>0$, therefore $\Re(q+\psi(\xi))>0$ in the strip $\Im \xi \in[-1,0]$ (see the proof of Lemma 3.5 in [12]). By applying Lemma 3.2, we conclude that $\phi_{q}^{+}$is holomorphic in a half-plane $\Im \xi>\omega_{-}$, for some $\omega_{-}<-1$, and hence, $\phi_{q}^{+}(D) e^{x}=$ $\phi_{q}^{+}(-i) e^{x}$ and $\phi_{q}^{+}(D) 1=1$ are well-defined. Thus, we can rewrite (4.8) as

$$
w_{j}(x)=w_{j 1}(x)+\phi_{q}^{+}(-i) e^{x}-(1+r \Delta) K,
$$

where $w_{j 1}(x):=\left(\phi_{q}^{+}(D) v_{j+1}\right)(x)$. We know from Theorem 3.1 that $\phi_{q}^{+}(\xi)$ (resp., $\left.\phi_{q}^{-}(\xi)\right)$ is the Fourier transform of an infinitely divisible distribution $P_{q}^{+}$(resp., $\left.P_{q}^{-}\right)$supported on $(-\infty, 0]$ (resp., $\left.[0,+\infty)\right)$, and in the case of an RLPE, $P_{q}^{ \pm}$has the density: $P_{q}^{ \pm}(d x)=k_{q}^{ \pm}(x) d x$, where $k_{q}^{ \pm}(x)=0, \forall \pm x>0$. It can be shown that $v_{j+1}(x) \rightarrow 0$ as $x \rightarrow+\infty$ (recall that $v_{j+1}(x)$ is an approximation to the put price at $t=t_{j+1}$ and the spot price of the underlying $S(t)=e^{x}$ ), therefore

$$
w_{j 1}(x)=\int_{x}^{+\infty} k_{q}^{+}(x-y) v_{j+1}(y) d y .
$$

Similarly, we can rewrite (4.7) as

$$
v_{j}(x)=K-e^{x}+(1+r \Delta)^{-1} v_{j 1}(x),
$$

where for $x \leq h_{j}, v_{j 1}(x)=0$, and for $x>h_{j}$,

$$
v_{j 1}(x)=\int_{h_{j}}^{x} k_{q}^{-}(x-y) w_{j}(y) d y .
$$

In the next Section, we consider the calculation of the integrals in (4.11)-(4.13) in the case of jump-diffusions, when the procedure is especially simple. 


\section{JUMP-DIFFUSION CASE}

5.1. Preliminary analysis for the two-jump case. Consider the process in Example 2.1 with $c_{ \pm}>0$; the factors in the Wiener-Hopf factorization formulas are calculated in Example 3.1. Represent $\phi_{q}^{ \pm}(\xi)$ in (3.14) and (3.15) as sums of simple fractions

$$
\begin{aligned}
\phi_{q}^{+}(\xi) & =\sum_{j=0,1} \frac{B_{j}^{+}(q)}{\beta_{j}^{+}-i \xi}, \\
\phi_{q}^{-}(\xi) & =\sum_{j=0,1} \frac{B_{j}^{-}(q)}{-\beta_{j}^{-}+i \xi},
\end{aligned}
$$

where

$$
B_{j}^{+}(q)=\frac{\beta_{j}^{+} \beta_{k}^{+}\left(\lambda_{-}+\beta_{j}^{+}\right)}{\lambda_{-}\left(\beta_{k}^{+}-\beta_{j}^{+}\right)}, \quad B_{j}^{-}(q)=\frac{-\beta_{j}^{-} \beta_{k}^{-}\left(\lambda_{+}+\beta_{j}^{-}\right)}{\lambda_{+}\left(\beta_{k}^{-}-\beta_{j}^{-}\right)},
$$

and $k \in\{0,1\}$ is determined by $k \neq j$. For $a>0$, functions $1 /(a-i \xi)$ and $1 /(a+i \xi)$ are Fourier transforms of functions $e^{a x} \mathbf{1}_{(-\infty, 0]}(x)$ and $e^{-a x} \mathbf{1}_{[0,+\infty)}(x)$, respectively. This means that $R_{a}^{+}:=(a-i D)^{-1}$ and $R_{a}^{-}:=(a+i D)^{-1}$ are convolution operators with kernels $e^{a x} \mathbf{1}_{(-\infty, 0]}(x)$ and $e^{-a x} \mathbf{1}_{[0,+\infty)}(x)$ :

$$
\begin{aligned}
& R_{a}^{+} u(x)=\int_{-\infty}^{0} e^{a y} u(x-y) d y=\int_{x}^{+\infty} e^{a(x-y)} u(y) d y \\
& R_{a}^{-} u(x)=\int_{0}^{+\infty} e^{-a y} u(x-y) d y=\int_{-\infty}^{x} e^{-a(x-y)} u(y) d y .
\end{aligned}
$$

By using (5.1) and (5.2), we can write (4.11) as

$$
w_{j 1}(x)=\sum_{l=0,1} B_{l}^{+}(q) \int_{x}^{+\infty} e^{\beta_{l}^{+}(x-y)} v_{j+1}(y) d y
$$

and (4.13) as

$$
v_{j 1}(x)=\sum_{l=0,1} B_{l}^{-}(q) \int_{h_{j}}^{x} e^{\beta_{l}^{-}(x-y)} w_{j}(y) d y .
$$

Notice that (4.13), hence, (5.5) are applied for $x>h_{j}$ only.

The following consideration shows that since the terminal payoff $v_{n}(x)=\left(K-e^{x}\right)_{+}$is a piece-wise exponential polynomial, the integrals in (5.4) and (5.5) are easy to compute, and they define piece-wise exponential polynomials. (An exponential polynomial is a function of 
the form

$$
u(x)=\sum_{l=0}^{m} c_{l} x^{l} e^{\gamma_{l} x},
$$

where $c_{l}$ and $\gamma_{l}$ are constants.) Clearly, for any $h \leq+\infty, a \in \mathbf{R}$, and any exponential polynomial $u$, the formula

$$
R_{a}^{+} u(x)=\int_{x}^{h} e^{a(x-y)} u(y) d y, \quad x<h,
$$

defines an exponential polynomial on $(-\infty, h)$ (in the case $h=+\infty, a$ must be greater than any $\gamma_{l}$ ). Similarly, for any $h>-\infty, a \in \mathbf{R}$, and any exponential polynomial $u$,

$$
R_{a}^{-} u(x)=\int_{h}^{x} e^{-a(x-y)} u(y) d y, \quad x>h,
$$

defines an exponential polynomial on $(h,+\infty)$. In each case, the coefficients and exponents can easily be calculated by using the method of indeterminate coefficients.

These two observations are implicitly used in the textbooks on linear ODE, of the first order, and the following interpretation of (5.4) and (5.5) may put the reader into a familiar picture:

$$
\begin{aligned}
& w_{j 1}(x)=\sum_{l=0,1} B_{l}^{+}(q) w_{j 1 l}(x), \\
& v_{j 1}(x)=\sum_{l=0,1} B_{l}^{-}(q) v_{j 1 l}(x),
\end{aligned}
$$

where

- $w_{j 1 l}$ is the solution to ODE

$$
w^{\prime}(x)=\beta_{l}^{+} w(x)+v_{j+1}(x), \quad \text { s.t. } w(+\infty)=0 ;
$$

- $v_{j 1 l}$ is the solution to ODE

$$
v^{\prime}(x)=\beta_{l}^{-} v(x)+w_{j}(x), \quad x>h_{j}, \quad \text { s.t. } v\left(h_{j}\right)=0 .
$$

Since the initial datum $v_{n}$ is given by exponential polynomials on two (semi-infinite) intervals $\left(-\infty, h_{n}\right]$ and $\left[h_{n},+\infty\right)$, namely, $v_{n}(x)=0, x \geq$ 0 , and $v_{n}(x)=1-e^{x}, x \leq 0$, we conclude that $w_{n-1,1, l}, l=0,1$, hence, $w_{n-1}$, are defined by exponential polynomials on the same intervals, and the early exercise boundary $h_{n-1}$ is the unique zero of $w_{n-1}$ on the left interval. Similarly, $v_{n-1,1, l}, l=0,1$, hence, $v_{n-1}$, are given by exponential polynomials on intervals $\left(-\infty, h_{n-1}\right],\left[h_{n-1}, h_{n}\right],\left[h_{n},+\infty\right)$. By iterating, we conclude that for $j=n-1, n-2, \ldots, w_{j, 1, l}, l=0,1$, and $w_{j}$ are given by exponential polynomials on $\left(-\infty, h_{j+1}\right],\left[h_{j+1}, h_{j+3}\right]$, 
$\ldots,\left[h_{n-1}, h_{n}\right],\left[h_{n},+\infty\right)$, and $v_{j, 1, l}, l=0,1$, and $v_{j}$ are given by exponential polynomials on $\left(-\infty, h_{j}\right],\left[h_{j}, h_{j+1}\right], \ldots,\left[h_{n-1}, h_{n}\right],\left[h_{n},+\infty\right)$. Notice that only exponentials $e^{\beta_{\alpha}^{ \pm} x}(\alpha=0,1), e^{x}$ and $e^{0}$ are involved, and the orders of polynomials on each step and interval can be computed quite easily. In particular, the exponential polynomials with factors $e^{x}$ and $e^{0}$ are always of order 0 , and the orders of exponential polynomials with factors $e^{\beta^{ \pm} x}(\alpha=0,1)$ grow not faster than by 1 on each time step (more accurate description of the orders can easily be made for each interval and time step). Further, on $\left[h_{n},+\infty\right), v_{j}$ and $w_{j, 1, l}$ are given by exponential polynomials with negative factors in exponents, and on $\left(-\infty, h_{j}\right], v_{j}(x)=K-e^{x}$. Therefore it is straightforward to write a program which computes coefficients of exponential polynomials $w_{j, 1, l}, l=0,1$, and $w_{j}$ on each interval (given coefficients of $v_{j+1}$ on the same intervals), and coefficients of exponential polynomials $v_{j, 1, l}, l=0,1$, and $v_{j}$ on each interval (given coefficients of $w_{j}$ on the same intervals). For $w_{j}$, first, the coefficients are calculated for the restriction on the rightmost interval $\left[h_{n},+\infty\right)$, and then we move step by step to the left using the method of indeterminate coefficients and the continuity of $w_{j, 1, l}, l=0,1$, at $h_{n}, h_{n-1}, \ldots, h_{j+1}$. When the coefficients of the restriction of $w_{j}$ on $\left(-\infty, h_{j+1}\right]$ are found, we solve the equation $w_{j}(x)=0$ on this interval $\left(w_{j}\right.$ changes the sign there), and denote the root by $h_{j}$. After that we define $v_{j}(x)=K-e^{x}$ on $\left(-\infty, h_{j}\right]$, compute coefficients of (the restrictions of) $v_{j, 1, l}, l=0,1$, and $v_{j}$ on $\left[h_{j}, h_{j+1}\right]$ by using the method of indeterminate coefficients and the continuity condition at $h_{n-j}$, and move step by step to the right.

The recurrent procedure of the calculation of coefficients of exponential polynomials is purely algebraic one: the method of indeterminate coefficients involves only the solution of linear systems of a simple structure, and it requires neither truncation of integrals nor discretization of the state space and numerical integration; in addition, on each step, the unique solution of an increasing function must be found, which can be done with the help of a simple numerical procedure. It can be shown that the total number of the coefficients of the exponential polynomials $v_{j}^{s}, j=n-1, n-2, \ldots, 0, s=j+1, j+2, \ldots, n+1$, is of order $2 n^{3} / 3$, and so their number and time of calculation is not large even for $n=100$, say. To be specific, the number of the coefficients of $v_{j}^{n+1}$ (resp., $\left.v_{j}^{s}, s \leq n\right)$ is $2(n-j)$ (resp, $\left.4(s-j)\right)$, and the total number is

$$
2 \sum_{j=0}^{n-1}(n-j)+4 \sum_{j=0}^{n-1} \sum_{s=j+1}^{n}(s-j)=\frac{2 n^{3}+9 n^{2}+7 n}{3} .
$$


5.2. Semi-explicit pricing procedure. At $j=n, n-1, \ldots$, we know optimal boundary points $h_{j}<h_{j+1}<\cdots<h_{n}=\ln K$, and $v_{j}$. On $\left[h_{n},+\infty\right), v_{j}$ coincides with an exponential polynomial of the form

$$
v_{j}^{n+1}(x)=\sum_{\alpha=0,1} e^{\beta_{\alpha}^{-} x} P_{j,-, \alpha}^{n+1}(x)
$$

(for $j=n$, it is zero); on $\left(-\infty, h_{j}\right], v_{j}$ coincides with $v_{j}^{j}(x)=K-e^{x}$, and on $\left[h_{s-1}, h_{s}\right], s=j+1, j+2, \ldots, n$, with an exponential polynomial of the form

$$
v_{j}^{s}(x)=v_{j}^{s, 0}+v_{j}^{s, 1} e^{x}+\sum_{\alpha=0,1} e^{\beta_{\alpha}^{-} x} P_{j,-, \alpha}^{n+1}(x)+\sum_{\alpha=0,1} e^{\beta_{\alpha}^{+} x} P_{j,+, \alpha}^{n+1}(x) .
$$

The recursive procedure for the calculation of the coefficients of $v_{j}^{s}$ is as follows. For $j=n, n-1, \ldots$ :

Step 1. Find functions $w_{j-1,1, \gamma}^{n+1}, \gamma=0,1$, in the form (5.8), by applying the method of indeterminate coefficients to an ODE

$$
w^{\prime}-\beta_{\gamma}^{+} w=v_{j}^{n+1} \text {, s.t. } w(+\infty)=0,
$$

and then set

$$
w_{j-1}^{n+1}(x)=\sum_{\gamma=0,1} B_{\gamma}^{+}(q) w_{j-1,1, \gamma}^{s}(x)+\phi_{q}^{+}(-i) e^{x}-(1+r \Delta) K .
$$

Step 2. For $s=n, n-1, \ldots, j$, find $w_{j-1,1, \gamma}^{s}, \gamma=0,1$, as a solution to the ODE

s.t.

$$
w^{\prime}-\beta_{\gamma}^{+} w=v_{j}^{s}
$$

$$
w\left(h_{s}\right)=w_{j-1,1, \gamma}^{s+1}\left(h_{s}\right)
$$

By using the method of the indeterminate coefficients, one can find all the coefficients of the exponential polynomial

$$
\begin{aligned}
w_{j-1,1, \gamma}^{s}(x)= & w_{j-1,1, \gamma}^{s, 0}+w_{j-1,1, \gamma}^{s, 1} e^{x} \\
& +\sum_{\alpha=0,1} e^{\beta_{\alpha}^{-} x} Q_{j,-, \gamma, \alpha}^{s}(x)+\sum_{\alpha=0,1} e^{\beta_{\alpha}^{+} x} Q_{j,+, \gamma, \alpha}^{s}(x),
\end{aligned}
$$

except for the zero-order term of $Q_{j,+, \gamma, \gamma}^{s}$, which is to be found afterwards, from the continuity condition (5.10). The coefficients of $w_{j-1,1, \gamma}^{s}, \gamma=0,1$, being found, one calculates the coefficients of

$$
w_{j-1}^{s}(x)=\sum_{\gamma=0,1} B_{\gamma}^{+}(q) w_{j-1,1 \gamma}(x)+\phi_{q}^{+}(-i) e^{x}-(1+r \Delta) K .
$$

Step 3. Find the unique root of the equation $w_{j-1}^{s}(x)=0$ on $\left(-\infty, h_{j}\right]$, and denote it by $h_{j-1}$. 
Step 4. Define $v_{j-1}^{j-1}$, the restriction of $v_{j-1}$ on $\left(-\infty, h_{j-1}\right]$, as $K-e^{x}$, and set $v_{j-1,1, \gamma}^{j-1}=0, \gamma=0,1$.

Step 5. For $s=j, j+1, \ldots, n+1$, find $v_{j-1,1, \gamma}^{s}, \gamma=0,1$, as a solution to the ODE

$$
v^{\prime}-\beta_{\gamma}^{-} v=w_{j-1}^{s},
$$

s.t.

$$
v\left(h_{s-1}\right)=v_{j-1,1, \gamma}^{s-1}\left(h_{s-1}\right) .
$$

By using the method of the indeterminate coefficients, one can find all the coefficients of the exponential polynomial

$$
\begin{aligned}
v_{j-1,1, \gamma}^{s}(x)= & v_{j-1,1, \gamma}^{s, 0}+v_{j-1,1, \gamma}^{s, 1} e^{x} \\
& +\sum_{\alpha=0,1} e^{\beta_{\alpha}^{-} x} P_{j,-, \gamma, \alpha}^{s}(x)+\sum_{\alpha=0,1} e^{\beta_{\alpha}^{+} x} P_{j,+, \gamma, \alpha}^{s}(x),
\end{aligned}
$$

except for the zero-order term of $P_{j,-, \gamma, \gamma}^{s}$, which is to be found afterwards, from the continuity condition (5.12). The coefficients of $v_{j-1,1, \gamma}^{s}, \gamma=0,1$, being found, one calculates the coefficients of

$$
v_{j-1}^{s}(x)=K-e^{x}+(1+r \Delta)^{-1} \sum_{\gamma=0,1} B_{\gamma}^{-}(q) v_{j-1,1, \gamma}^{s}(x) .
$$

Step 6. In the formula for $v_{j-1}^{n+1}$, set all the coefficients at the exponential polynomials with non-negative exponents equal to 0 (if all the numerical calculation were exact, these coefficients would have been zero but due to computational errors, they may be non-zero albeit small).

Thus, we have found $v_{j-1}$ and $h_{j-1}$, and can repeat the steps above with $j-1$ instead of $j$, etc.

5.3. Numerical examples. In the first example shown on Figure 1, we take $K=T=1$, and $n=20$. We fix the instantaneous variance $m_{2}=\psi^{\prime \prime}(0)=0.3, r=0.05, T=1$, and $c_{ \pm}=1, \lambda_{-}=-20$, and study how the discretized early exercise boundary depends on $\lambda_{+}$. Recall that $\lambda_{+}$characterizes the relative intensity of large negative jumps, and that the last parameter, $b$, is determined from the condition $r+\psi(-i)=0$. The smaller the $\lambda_{+}$is (and hence, the larger the relative intensity of big negative jumps w.r.t. small ones), the higher the early exercise boundary is. This seemingly counterintuitive result agrees with optimal exercise prices $h_{*}=0.2061,0.1736,0.1646$ for the perpetual put, and shows that among processes with the same instantaneous variance, the ones with large negative jumps may lead to higher early exercise price. The next example shown on Figure 2 demonstrates that if we keep the volatility of the Gaussian component and positive jump component fixed and increase the intensity of negative jumps then the optimal 
exercise prise decreases as one should expect; the optimal exercise price for the perpetual put also decreases: $h_{*}=0.2419,0.2290,0.2091$.

5.4. Brownian motion case and one-jump case. The modification of the algorithm for the case when one or both of the $c_{ \pm}$in (2.5) are zero is evident: if both $c_{ \pm}=0$, then there are only two roots $\beta^{ \pm}(q)$, and in (5.4) and (5.5), there is only one term on the RHS. If one of the $c_{ \pm}$is 0 , and the other is not, then there exist 3 roots, and two terms in (5.4) or (5.5).

5.5. Multi-jump case. Consider the multi-jump generalization of (2.5):

$F(d x)=\sum_{k=1}^{K} c_{k,+} \lambda_{k,+} e^{\lambda_{k,+} x} \mathbf{1}_{(-\infty, 0)}(x) d x-\sum_{l=1}^{L} c_{l,-} \lambda_{l,-} e^{\lambda_{l,-} x} \mathbf{1}_{(0,+\infty)}(x) d x$,

where $\lambda_{k,+}>0, \lambda_{l,-}<-1$ and $c_{j, \pm}>0$. Then

$$
\psi(\xi)=\frac{\sigma^{2}}{2} \xi^{2}-i b \xi+\sum_{k=1}^{K} \frac{i c_{k,+} \xi}{\lambda_{k,+}+i \xi}+\sum_{l=1}^{L} \frac{i c_{l,-} \xi}{\lambda_{l,-}+i \xi}
$$

where $\sigma^{2}>0$ and $b \in \mathbf{R}$ are the variance and drift of the Gaussian component. To factorize $1+\Delta(r+\psi(\xi))$, it suffices to find the roots of equation (3.12). In terms of the variable $\beta=i \xi$, equation (3.12) assumes the form

$$
q-b \beta-\frac{\sigma^{2}}{2} \beta^{2}+\sum_{k=1}^{K} \frac{c_{k,+} \beta}{\lambda_{k,+}+\beta}+\sum_{l=1}^{L} \frac{c_{l,-} \beta}{\lambda_{l,-}+\beta}=0
$$

Equation (5.16) has $1+L$ positive roots $\beta_{l}^{+}=\beta_{l}^{+}(q), l=0, \ldots, L$, and $1+K$ negative roots $\beta_{k}^{-}=\beta_{k}^{-}(q), k=0, \ldots, K$, which are separated by 0 and the poles $-\lambda_{ \pm, j}$; hence, they can be calculated easily with high precision. By using (3.10) and (3.11), we obtain

$$
\begin{aligned}
\phi_{q}^{+}(\xi) & =\prod_{l=0}^{L} \frac{\beta_{l}^{+}}{\beta_{l}^{+}-i \xi} \prod_{l=1}^{L} \frac{-\lambda_{l,-}-i \xi}{-\lambda_{l,-}} \\
\phi_{q}^{-}(\xi) & =\prod_{k=0}^{K} \frac{-\beta_{k}^{-}}{-\beta_{k}^{-}+i \xi} \prod_{k=1}^{K} \frac{\lambda_{k,+}+i \xi}{\lambda_{k,+}} .
\end{aligned}
$$

Represent $\phi_{q}^{ \pm}(\xi)$ as sums of simple fractions

$$
\phi_{q}^{+}(\xi)=\sum_{l=0}^{L} \frac{B_{l}^{+}(q)}{\beta_{l}^{+}-i \xi}, \quad \phi_{q}^{-}(\xi)=\sum_{k=0}^{K} \frac{B_{k}^{-}(q)}{-\beta_{k}^{-}+i \xi}
$$




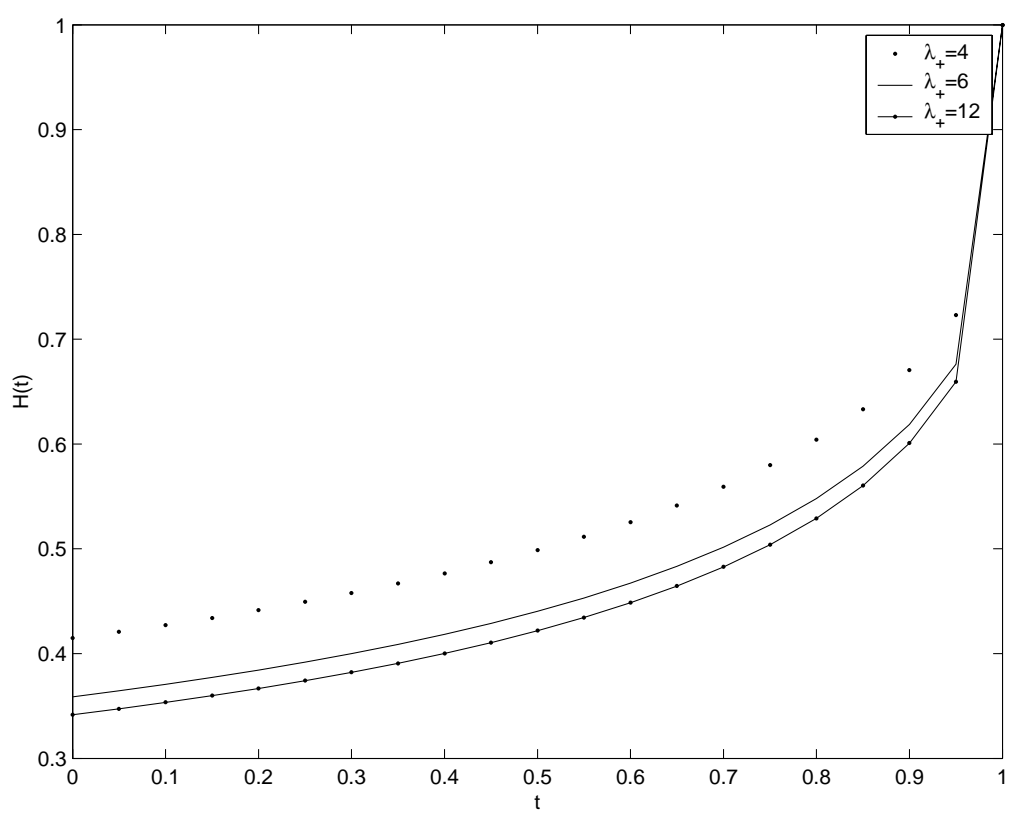

FIGURE 1. Early exercise boundary: instantaneous variance is fixed. Parameters: $K=1, T=1, n=20, r=$ $0.05, m_{2}=0.3, c_{+}=c_{-}=1, \lambda_{-}=-20$.

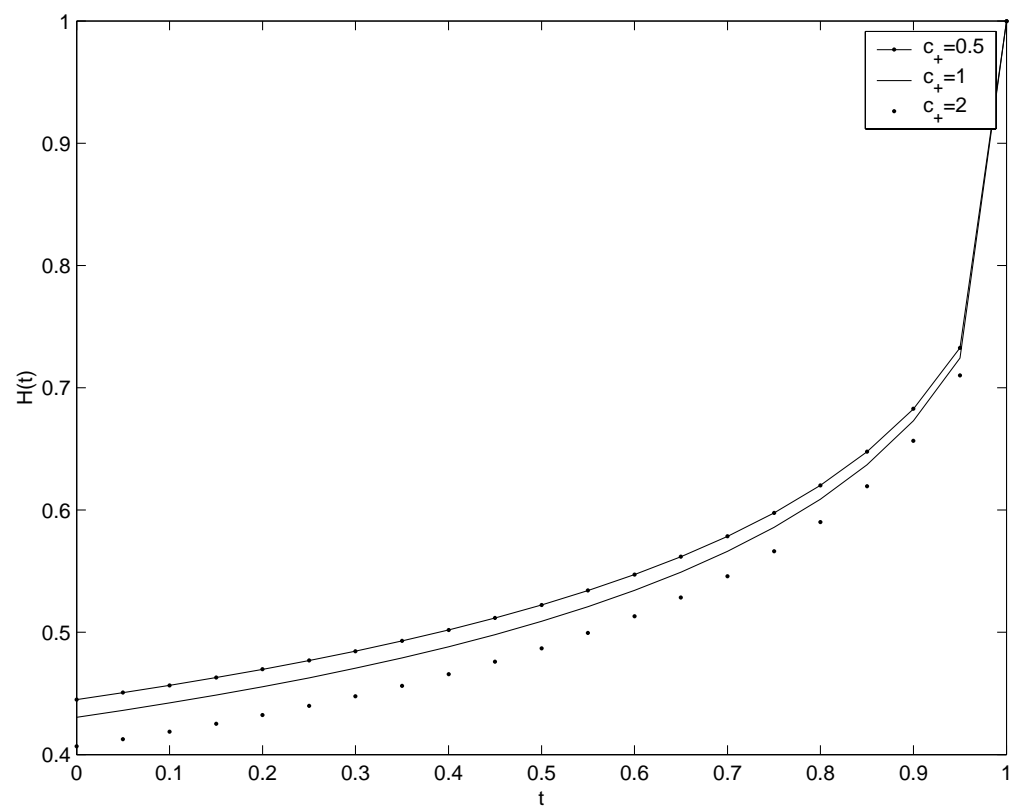

FiguRE 2. Early exercise boundary: volatility of the Gaussian component is fixed. Parameters: $K=1, T=$ $1, n=20, r=0.05, \sigma^{2}=0.3, c_{-}=1, \lambda_{+}=10, \lambda_{-}=-20$. 
where

$$
\begin{aligned}
& B_{m}^{+}(q)=\beta_{m}^{+} \prod_{l=0, \ldots, L, l \neq m} \frac{\beta_{l}^{+}}{\beta_{l}^{+}-\beta_{m}^{+}} \prod_{l=1, \ldots, L} \frac{-\lambda_{l,-}-\beta_{m}^{+}}{-\lambda_{l,-}}, \\
& B_{m}^{-}(q)=-\beta_{m}^{-} \prod_{k=0, \ldots, K, k \neq m} \frac{\beta_{k}^{-}}{\beta_{k}^{-}-\beta_{m}^{-}} \prod_{k=1, \ldots, K} \frac{-\lambda_{k,+}-\beta_{m}^{-}}{-\lambda_{k,+}} .
\end{aligned}
$$

By using (5.19), we can write (4.11) and (4.13) as

$$
\begin{aligned}
w_{j 1}(x) & =\sum_{l=0}^{L} B_{l}^{+}(q) \int_{x}^{+\infty} e^{\beta_{l}^{+}(x-y)} v_{j+1}(y) d y, \\
v_{j 1}(x) & =\sum_{k=0}^{K} B_{k}^{-}(q) \int_{h_{j}}^{x} e^{\beta_{k}^{-}(x-y)} w_{j}(y) d y .
\end{aligned}
$$

(Recall that (4.13), hence, (5.20) is applied for $x>h_{j}$ only). Now we can proceed further exactly as in the two-jump case, and obtain a pricing procedure, which takes as much time as calculation of solutions to $(2+L+K) n$ first-order linear ODE with constant coefficients and exponential polynomials as free terms.

\section{Detailed algorithm, the case of a general RLPE}

6.1. General analysis. In general case, we have to calculate the densities $k_{q}^{ \pm}$for $x_{l}$ from a sufficiently fine grid (after $\Delta>0$ is chosen). Next, the equations (4.10) and (4.11) can be used to calculate values $w_{j}(x)$ on an appropriate grid provided we know how to truncate the upper limit of the integration in (4.11), call it $A$; and the same limit determines the interval $\left[h_{j}, A\right]$, on which values of $v_{j 1}$ and $v_{j}$ need to be computed. The $A$ can be found with the help of the following lemma, which shows that $v_{j}(x)$ exponentially decays as $x \rightarrow+\infty$, and gives the rate of the decay. Without loss of generality, we normalize the strike price to one: $K=1$.

Lemma 6.1. Let $\omega_{+}>0$ be from estimate (3.5). Then for any $\omega \in$ $\left(0, \omega_{+}\right)$, and $j=n, n-1, \ldots$, there exists $C_{\omega, j}$ such that

$$
v_{j}(x) \leq C_{\omega, j} e^{-\omega x}, \quad \forall x .
$$

Proof. For simplicity, we assume that $\omega_{+}$is greater than 1 and so large that $r+\psi\left(i \omega_{+}\right)<0$; then $\omega \in\left(1, \omega_{+}\right)$can be chosen so that $r+\psi(i \omega)<$ 0 as well, and we make such a choice. The general case can be treated similarly only the formulas for the $C_{\omega, j}$ below become more complex. 
We prove (6.1) by induction. For $j=n, v_{n}(x)=\left(1-e^{x}\right)_{+}$, and hence, (6.1) holds with $C_{\omega, n}=1$. Suppose that (6.1) has been proven for $j=n, n-1, \ldots, m+1$. By using (4.12) and (4.8), we obtain

$$
\begin{aligned}
v_{m}= & 1-e^{x}+(1+r \Delta)^{-1} \phi_{q}^{-}(D) \mathbf{1}_{\left[h_{m},+\infty\right)} w_{m} \\
= & 1-e^{x}+(1+r \Delta)^{-1} \phi_{q}^{-}(D) \mathbf{1}_{\left[h_{m},+\infty\right)}\left(\phi_{q}^{+}(-i) e^{x}-(1+r \Delta)\right) \\
& +(1+r \Delta)^{-1} \phi_{q}^{-}(D) \mathbf{1}_{\left[h_{m},+\infty\right)} \phi_{q}^{+}(D) v_{m+1} \\
= & 1-e^{x}+(1+r \Delta)^{-1} \phi_{q}^{-}(D)\left(\phi_{q}^{+}(-i) e^{x}-(1+r \Delta)\right) \\
& -(1+r \Delta)^{-1} \phi_{q}^{-}(D) \mathbf{1}_{\left(-\infty, h_{m}\right]}\left(\phi_{q}^{+}(-i) e^{x}-(1+r \Delta)\right) \\
& +(1+r \Delta)^{-1} \phi_{q}^{-}(D) \mathbf{1}_{\left[h_{m},+\infty\right)} \phi_{q}^{+}(D) v_{m+1} .
\end{aligned}
$$

Since

$(1+r \Delta)^{-1} \phi_{q}^{-}(-i) \phi_{q}^{+}(-i)=(1+r \Delta)^{-1}(1+\Delta(r+\psi(-i)))^{-1}(1+r \Delta)=1$,

the first three terms on the RHS of the formula for $v_{m}$ cancel out, and we obtain

$$
v_{m}=(1+r \Delta)^{-1} \phi_{q}^{-}(D) w_{m 2}
$$

where

$$
w_{m 2}=\left((1+r \Delta)-\phi_{q}^{+}(-i) e^{x}\right) \mathbf{1}_{\left(-\infty, h_{m}\right]}+\mathbf{1}_{\left[h_{m},+\infty\right)} \phi_{q}^{+}(D) v_{m+1} .
$$

Since $h_{m}<\ln 1=0$, and

$$
e^{\omega x} \phi_{q}^{ \pm}(D) e^{-\omega x}=\phi_{q}^{ \pm}(D+i \omega),
$$

we have

$$
\begin{aligned}
e^{\omega x}\left|w_{m 2}(x)\right| & \leq \max \left\{(1+r \Delta), \phi_{q}^{+}(-i),\left(\phi_{q}^{+}(D+i \omega) e^{\omega \cdot} v_{m+1}\right)(x)\right\} \\
& \leq \max \left\{(1+r \Delta), \phi_{q}^{+}(-i), C_{\omega, m+1} \phi_{q}^{+}(i \omega)\right\}
\end{aligned}
$$

(the last estimate follows from (6.1) with $j=m+1$ ). By using (6.2) with the '-' sign, we continue:

$$
\begin{aligned}
e^{\omega x} v_{m}(x) & =(1+r \Delta)^{-1} \phi_{q}^{-}(D+i \omega) e^{\omega x} w_{m 2}(x) \\
& \leq(1+r \Delta)^{-1} \phi_{q}^{-}(i \omega) \max \left\{(1+r \Delta), \phi_{q}^{+}(-i), C_{\omega, m+1} \phi_{q}^{+}(i \omega)\right\} .
\end{aligned}
$$

Since

$$
(1+r \Delta)^{-1} \phi_{q}^{-}(i \omega) \phi_{q}^{+}(i \omega)=(1+\Delta(r+\psi(i \omega)))^{-1},
$$

we conclude that for $j=m$, (6.1) holds with

$$
C_{\omega, m} \leq \max \left\{\phi_{q}^{-}(i \omega), \frac{\phi_{q}^{-}(i \omega) \phi_{q}^{+}(-i)}{1+r \Delta}, \frac{C_{\omega, m+1}}{1+\Delta(r+\psi(i \omega))}\right\} .
$$


To apply estimate (6.1), it is desirable that the constant in the RHS be independent of $j$, and under assumption $r+\psi(i \omega)<0$, this can be achieved easily. Since $C_{\omega, n}=1$, we can simplify formula for $C_{\omega, j}$, $j \leq n-1$ :

$$
C_{\omega, j}=(1+\Delta(r+\psi(i \omega)))^{-n+j+1} C_{\omega}^{0}(\Delta),
$$

where

$C_{\omega}^{0}(\Delta)=\max \left\{\phi_{q}^{-}(i \omega),(1+r \Delta)^{-1} \phi_{q}^{-}(i \omega) \phi_{q}^{+}(-i),(1+\Delta(r+\psi(i \omega)))^{-1}\right\}$.

Since $n=T / \Delta$, and $r+\psi(i \omega)<0$, we have

$$
(1+\Delta(r+\psi(i \omega)))^{-n+j+1}<e^{-T(r+\psi(i \omega))},
$$

therefore (6.1) simplifies:

$$
v_{j}(x) \leq C_{\omega}^{0}(\Delta) e^{-T(r+\psi(i \omega))} e^{-\omega x}, \quad \forall x .
$$

Finally, from (6.4), it is easy to deduce that for $\omega$ fixed, $C_{\omega}^{0}(\Delta) \rightarrow 1$ as $\Delta \rightarrow 0$, therefore for small $\Delta$, the following simpler estimate may be used:

$$
v_{j}(x) \leq e^{-T(r+\psi(i \omega))} e^{-\omega x}, \quad \forall x
$$

6.2. Control of the truncation error for fixed $n$. In many empirical studies, the left tail is not very fat, that is, $\lambda_{+}$is large; then typically, $r+\psi\left(i\left(\lambda_{+}-0\right)\right)<0$. Further, from (3.5), it is easily seen that $\omega_{+}=\omega_{+}(\Delta) \rightarrow \lambda_{+}$as $\Delta \rightarrow 0$, therefore if $\Delta$ is small enough, then we can take $\omega=\lambda_{+}-1$, and apply estimate (6.6) (or more accurate estimate (6.5)). If we truncate the upper limit in integrals (4.11):

$$
w_{j 1}(x)=\int_{x}^{A} k_{q}^{+}(x-y) v_{j+1}(y) d y
$$

then each integration may yield an error $e^{-T(r+\psi(i \omega))} e^{-\omega A}$. We have to calculate $n$ integrals (4.11), therefore the cumulative error of the truncation is $n e^{-T(r+\psi(i \omega))} e^{-\omega A}$, and if we wish that the cumulative truncation error be less than $\epsilon>0$, we can find $A$ from the following condition:

$$
A>-\omega^{-1}[\ln [\epsilon / n]+T(r+\psi(i \omega))] .
$$

More accurate estimate is

$$
A>-\omega^{-1}\left[\ln \left[\epsilon /\left(n C_{\omega}^{0}(\Delta)\right)\right]+T(r+\psi(i \omega))\right] .
$$


6.3. Algorithm. We normalize $K=1$, fix $n$, the number of subintervals of $[0, T]$, and $\epsilon$, the truncation error, and assume that $\omega$ and $A$ can be chosen as indicated above. Assume that $A>0$; this is always the case if $\epsilon$ is small. Fix $\delta>0$, the step for a grid in the state space, choose an integer $N_{1} \geq A / \delta$, and construct $x_{l}=\left(N_{1}-l\right) \delta, l=0,1, \ldots, N$, where $N$ will be chosen below. For small $\Delta$ and $x$ in a neighborhood of 0 , the probability density $p_{\Delta}(x)$ of an RLPE of order $\nu$ behaves like the one of the stable Lévy process of index $\nu$. Therefore on the basis of scaling considerations, for large $n$, it is advisable to choose $\delta$ of order $\Delta^{1 / \nu}$. Otherwise $\delta$ should be chosen to make the error of numerical integration below as small as desired. After that we

1. calculate $q=\Delta^{-1}+r$ and $\phi_{q}^{ \pm}(-i)$;

2. compute $h_{*}=\ln \left[\phi_{r}^{-}(-i)\right]$, the optimal exercise log-price of the perpetual American put (see $[9,10]$ and Chapter 5 in [12]), and choose an integer $N$ so that $N \geq N_{1}-h_{*} / \delta$;

3 . for $s=0,1, \ldots, N$, calculate and store values $e_{s}=\exp \left[\left(N_{1}-s\right) \delta\right]$, and

$$
\begin{gathered}
k_{q, s}^{+}:=k_{q}^{+}(-s \delta)=(2 \pi)^{-1} \int_{-\infty}^{+\infty} e^{-i s \delta \xi} \phi_{q}^{+}(\xi) d \xi \\
k_{q, s}^{-}:=k_{q}^{-}(s \delta)=(2 \pi)^{-1} \int_{-\infty}^{+\infty} e^{i s \delta \xi} \phi_{q}^{-}(\xi) d \xi
\end{gathered}
$$

In the case of jump-diffusions, we can calculate $k_{q, s}^{-}$explicitly; in the general case, the Fast Fourier Transform can be used;

4. for $j=n$, set $h_{j}=0$, and define $v_{n, l}=0, l=0,1, \ldots, N_{1}-1$, and $l_{n}=N_{1}$

5. in the loop for $j=n-1, n-2, \ldots, 0$,

a) by applying a numerical integration procedure to (4.11) (say, the trapezoid rule or Simpson's rule), calculate approximations $w_{j 1, l}$ to $w_{j 1}\left(\left(N_{1}-l\right) \delta\right), l=0,1, \ldots$ Here the stored values $k_{q, s}^{+}, s=0,1, \ldots, N$, and $v_{j+1, s}, s=0,1, \ldots, l_{j}-1$, should be used, and when $v_{j+1, s}$ for $s \geq l_{j}$ are needed, one uses formula $v_{j+1, s}=1-e_{s}$. Then set

$$
w_{j, l}=w_{j 1, l}+\phi_{q}^{+}(-i) e_{l}-(1+r \Delta) .
$$

When $w_{j, l} \leq 0$, set $l_{j}=l$, stop the loop in $l$, and find $h_{j}$ by linear interpolation:

$$
h_{j}=\delta\left[N_{1}-l_{j}+w_{j, l_{j}} /\left(w_{j, l_{j}}-w_{j, l_{j}-1}\right)\right]
$$

(higher order interpolation can be used as well);

b) by applying a numerical integration procedure to (4.13), calculate approximations $v_{j 1, l}$ to $v_{j 1}(A-l \delta), l=l_{j}-1, \ldots, 0$. Here the stored 
values $k_{q, l-s}^{-}, s=0,1, \ldots, N$, and $w_{j, s}=w_{j}\left(\left(N_{1}-s\right) \delta\right), s=0,1, \ldots, l_{j}-$ $1, w_{j}\left(h_{j}\right)=0$ have to be used. Then set

$$
v_{j, l}=1-e_{l}+(1+r \Delta)^{-1} v_{j 1, l}
$$

as an approximation for the put price $v_{j}\left(\left(N_{1}-l\right) \delta\right), l<l_{j}$.

Remark. Near the early exercise boundary, derivatives of the put price, of order 3 and higher, are large, and therefore, the integrals over a small neighborhood of the boundary should be integrated by using the trapezoid rule; farther from the boundary, the Simpson rule can be used.

\section{Conclusion}

We considered the pricing problem for the American put with a finite time horizon, for wide classes of Lévy processes. By using time discretization (method of lines or equivalently, Carr's randomization), we reduced the problem to a series of free boundary problems on the line. We solved these problem by using the Wiener-Hopf factorization, and derived explicit pricing procedure, which gives the early exercise boundary and option prices for all strikes and time to maturity from interval $[0, T]$. In the case of jump-diffusions with $K$ jump components, the procedure is simplified further, and the result is the pricing scheme, which involves the recurrent integration of $(2+K) n$ linear ODE of the first order with constant coefficient, and piece-wise exponential polynomials as free terms (the method of indeterminate coefficients), and finding zeroes of some of these polynomials on intervals, where the zero exists and is unique. The coefficients of the polynomials and zeroes can be calculated easily, and with the high precision. The zeroes define the discretized early exercise boundary, and the coefficients can be used to compute the rational put price. The pricing procedure can be made faster by means of Richardson extrapolation scheme, as in the Gaussian case (see [13]). Numerical results are presented to demonstrate the dependence of the early exercise boundary and prices on the jump component.

\section{REFERENCES}

[1] G. Barles, J. Burdeau, M. Romano, and N. Samsoen, Critical stock price near expiration, Mathem. Finance 5 (1995), 77-95.

[2] O. E. BARNDORFF-NiElsen, Exponentially decreasing distributions for the logarithm of particle size, Proc. Roy. Soc. London. Ser. A, 353 (1977), pp. 401419.

[3] O. E. Barndorff-Nielsen, Processes of Normal Inverse Gaussian Type, Finance and Stochastics, 2 (1998), pp. 41-68. 
[4] O. E. Barndorff-Nielsen And W. Jiang, An initial analysis of some German stock price series, Working Paper Series, 15, Aarhus: CAF Univ. of Aarhus/Aarhus School of Business (1998).

[5] O. E. BARndorfF-Nielsen And S. LeVEndorskiǏ, Feller Processes of Normal Inverse Gaussian type, Quantitative Finance 1 (2001), pp. 318-331.

[6] O. E. BARndorfF-Nielsen And N. Shephard, Normal modified stable processes, forthcoming in Theory of Probability and Mathematical Statistics.

[7] J-P. Bouchaud And M. Potters, Theory of Financial Risk. Paris, AléaSaclay, Eurolles, 1997.

[8] S. I. Boyarchenko And S. Z. LeVEndorskiǏ, Generalizations of the BlackScholes equation for truncated Lévy processes, Working Paper (1999).

[9] S. I. BoyARChEnko And S. Z. LeVEndorskiǏ, Option pricing for truncated Lévy processes, Intern. Journ. Theor. and Appl. Finance, 3:3 (2000), pp. 549552 .

[10] S. I. Boyarchenko And S. Z. LevendorskiǏ, Perpetual American options under Lévy processes, SIAM Journ. Control and Optimization (2002), 40: 1663-1696.

[11] S. I. BoyARChEnko AND S. Z. LeVEndorskiǏ, Barrier options and touchand-out options under regular Lévy processes of exponential type, Annals of Appl. Probability (2002), forthcoming

[12] S. I. Boyarchenko And S. Z. LevendorskiǏ, Non-Gaussian Merton-BlackScholes theory, World Scientific, New Jercey, London, Singapore, Hong Kong, 2002

[13] P. CARR, Randomization and the American put, Review of Financial Studies 11:3 (1998), pp. 597-626.

[14] P. CARr, AND D. FAGUet, Fast accurate valuation of American options, Working paper, Cornell University (1994).

[15] P. Carr, H. Geman, D.B. Madan, M. Yor, The fine structure of asset returns: an empirical investigation, Journal of Business, 75 (2001), 305-332.

[16] E. Clément, D. Lamberton, And P.Protter, An analysis of a least squares regression method for American option pricing, Finance and Stochastics, 6 (2002), pp. 449-471.

[17] R. Cont, M. Potters And J.-P. Bouchaud, Scaling in stock market data: stable laws and beyond, In Scale Invariance and beyond (Proceedings of the CNRS Workshop on Scale Invariance, Les Houches, March 1997), B. Dubrulle, F. Graner, and D. Sornette, eds., Springer, Berlin, pp. 75-85, 1997.

[18] F. Delbaen And W. Schachermayer, A general version of the fundamental theorem of asset pricing, Math. Annalen, 300 (1994), pp. 463-520.

[19] E. EBerlein AND U. Keller, Hyperbolic distributions in finance, Bernoulli, 1 (1995), pp. 281-299.

[20] E. Eberlein, U. Keller And K. Prause, New insights into smile, mispricing and value at risk: The hyperbolic model, Journ. of Business, 71 (1998), pp. 371-406.

[21] E. EBerlein And S. RAIBLe, Term structure models driven by general Lévy processes, Math. Finance, 9 (1999), pp. 31-53.

[22] B.Hilberink And L.C.G. Rogers, Optimal capital structure and endogenous default, Finance and Stochastics, 6, (2002), 237-263. 
[23] I. Karatzas, And S. E. Shreve, Methods of Mathematical Finance, Springer-Verlag, Berlin Heidelberg New York, 1998

[24] I. Koponen, Analytic approach to the problem of convergence of truncated Lévy flights towards the Gaussian stochastic process, Phys. Rev. E, 52 (1995), pp. 1197-1199.

[25] Y.-K. KwoK, Mathematical Models of Financial Derivatives, Springer-Verlag, Berlin Heidelberg New York, 1998.

[26] D. Lamberton, Critical price for an American option near maturity, in Seminar on Stochastic Analysis, Random Fields and Applications, E. Bolthausen, M. Dozzi, F. Russo, eds. Birkhaüser, Boston Basel Berlin, 1995, pp. 353-358.

[27] F.A. Longstaff, E.S. Schwartz, Valuing American options by simulation: a simple least-squares approach, Rev. Financial Stud. 14 (2001), 113-148.

[28] D. B. Madan, P. Carr And E. C. Chang, The variance Gamma process and option pricing, European Finance Review, 2 (1998), pp. 79-105.

[29] R. N. Mantegna And H.E. Stanley, Stochastic process with ultraslow convergence to a gaussian: the truncated Lévy flight, Phys.Rev.Lett., 73 (1994), pp. 2946-2949.

[30] R. N. Mantegna and H.E. Stanley, Physics investigation of financial markets, Proceedings of the International School of Physics "Enrico Fermi", Course CXXXIV, F.Mallamace and H.E.Stanley, eds., IOS Press, Amsterdam, 1997, pp. 473-489.

[31] A. Matacz, Financial modeling and option theory with the Truncated Levy Process, Intern. Journ. Theor. and Appl. Finance, 3:1 (2000), pp. 143-160.

[32] M. Musiela, AND M.RutKowski, Martingale methods in financial modelling, Springer-Verlag, Berlin Heidelberg New York, 1997.

[33] L.C.G. Rogers And D. Williams, Diffusions, Markov Processes and Martingales, Volume 1: Foundations, Cambridge University Press, 2000.

[34] K. Sato, Lévy processes and infinitely divisible distributions, Cambridge University Press, Cambridge, 1999.

[35] N. WIENER, AND E. Hopf, Über eine Klasse singulärer Integralgleichungen, Sibtzer. Deutsch. Akad. Wiss. Berlin, Kl. Math. Phys. Tech. 1931, pp. 696-706.

[36] P. Wilmott, J. N. Dewynne, and S. Howison, Option pricing: Mathematical models and computation, Oxford Financial Press, Oxford, 1993. 